\title{
A Novel Bursting Mechanism of Type A Neurons in Injured Dorsal Root Ganglia
}

\author{
Z. Jian ${ }^{a}$ J.L. Xing ${ }^{b}$ G.S. Yang ${ }^{c}$ S.J. Hu ${ }^{b}$ \\ aDepartment of Physics; b Institute of Neuroscience and cDepartment of Electronics, \\ The Fourth Military Medical University, Xi'an, PR China
}

\section{Key Words}

Dorsal root ganglion - Elliptic bursting · Spontaneous discharge $\cdot$ Unstable periodic orbits

\begin{abstract}
Using intracellular recording in vivo, the bursting behaviors were investigated in the neurons of chronically compressed dorsal root ganglia of the adult rat. In most cases, the first spike of a burst emerged from amplitudeincreasing damped subthreshold membrane potential oscillation (SMPO) and the discharge terminated by an amplitude-decreasing damped SMPO. The rhythms of these bursting behaviors are all irregular. Since some researchers found that the stochastic dynamics can also produce similar bursting pattern, the deterministic dynamics of interevent interval (IEI) series obtained from raw membrane potential recording was detected by extraction of the hierarchy of unstable periodic orbits (UPOs) in the windowed IEI series. The results showed a number of statistically significant UPOs of order-one, order-two, and order-three. These orbits form a complex but predictable lattice of regions in which the dynamics of the bursting occurrence is deterministic. Based on a complete classification scheme, the investigated bursting can be depicted by the elliptic bursting dynamics. The significance of the finding that a neuron in the injured dorsal root ganglion has such dynamics is also
\end{abstract} discussed.

Copyright $(2004$ S. Karger AG, Basel

\section{Introduction}

Bursting behavior in a neuron is a recurrent transition between a quiescent state and a repetitive firing state. When the transition to repetitive spiking occurs via a subcritical Andronov-Hopf bifurcation and the transition to the quiescent state occurs via fold limit cycle bifurcation, the bursting behavior is said to be of elliptic type [1]. A neuron having such dynamics owns a very interesting coding rule largely different from that of an integrate-andfire neuron that performs temporal integration of the incoming pulse trains. According to some simulation results [2], the dynamic responsiveness of such a neuron will act as a resonate-and-fire neuron that selectively interacts with other neurons.

Based on this bursting mechanism, some experiential criterions were used to identify this type of bursting [3]. It is characterized by some topological geometric features of voltage oscillations, i.e. the oscillation of membrane potential normally evolves from growing subthreshold membrane potential oscillation (SMPO) with full-amplitude spikes and spike frequency may not decline at burst termination. Recently, a novel type of spontaneous bursting behavior in neurons of injured dorsal root ganglion (DRG) has been reported [4-9]. In view of a complete classification scheme of deterministic bursting dynamics [10], these discharges are similar to elliptic bursting. However, previous research did not firstly examine whether these bursting discharges come from deterministic dy-

Prof. S.J. Hu

Institute of Neuroscience

The Fourth Military Medical University

No.17 West Chang-le Road, Xi'an 710032 (PR China)

Tel. +86 29 3374590, Fax +86293246270, E-Mail sjhu@fmmu.edu.cn 
namics [3]. Some mathematical simulation results and experimental investigations showed that similar bursting pattern can also be induced from stochastic neuronal dynamics [11-13]. Thus, what the underlying dynamics of this bursting pattern generated in neurons of injured $\mathrm{DRG}$ are, is still an open question.

Based on the biological model of chronic compression of DRG [14,15], the bursting behaviors similar to those of elliptic type were recorded and extraction of unstable periodic orbits (UPOs) was applied to detect the deterministic dynamics of the spontaneous discharge so as to exclude the possibility of stochastic dynamics.

\section{Materials and Methods}

\section{Experimental Model}

By using a previously described method [14, 15], the experimental model was prepared on adult Sprague-Dawley rats of both sexes weighing 150-250 g. After anesthetization with sodium pentobarbital $(40 \mathrm{mg} / \mathrm{kg}$, i.p.), the L5 intervertebral foramen on one side was exposed under sterile conditions and an L-shaped stainless steel rod (about $4 \mathrm{~mm}$ in length and $0.5-0.8 \mathrm{~mm}$ in diameter) was inserted into the foramen to produce a steady compression on L5 DRG. The laminectomy from L4 to L6 was performed after 2-8 days, and a small pool was made of skin flaps and muscle under chloralose-urethane anesthesia. The L5 DRG was fully exposed and the rod carefully removed. The sciatic nerve was transected at mid-thigh and a bipolar stimulating electrode was placed on the central end of transected sciatic nerve. The capsule on L5 DRG was slit open with fine forceps to expose the DRG. The pool was filled with artificial cerebrospinal fluid to cover the exposed tissue. The animal was fixed on the stereotactic frame and immobilized with $4 \mathrm{mg} / \mathrm{kg}$ curare under artificial respiration in order to minimize reflex movements. Intracellular recording was made using 50-100 $\mathrm{M} \Omega$ resistance microelectrode filled with $3 M$ potassium acetate solution. The recording was displayed on a memory oscilloscope and the data were stored for analysis. DRG neurons were categorized by the shape of spikes recorded and conduction velocity $(\mathrm{CV})$ of the nerve fiber measured by electrical stimulation of the sciatic nerve. Cells were designated as type A neurons, according to the criterion of $\mathrm{CV}>2 \mathrm{~m} / \mathrm{s}$ or no inflection on the falling phase of the spike and/or spike width $<2 \mathrm{~ms}$ at one-half peak-to-peak amplitude. Cell penetrations yielding relatively stable membrane potential more negative than $-40 \mathrm{mV}$ for $>3$ min were investigated further.

\section{Extraction of UPO}

The bursting event is defined as the longest sequences of spikes with all interspike intervals (ISIs) less than or equal to a certain discriminating threshold which was selected for each record [16]. According to the above definition illustrated in figure $2 \mathrm{a}$, the ISI series were processed to extract bursting events and the interevent interval (IEI) series were used for analysis.

Our basic assumption is that there exists a significant deterministic component within the seemingly noisy bursting discharge of neurons, and UPOs can be used to characterize the system's deterministic dynamics. A UPOs extraction method developed by So et al. [17-
19] was used in our study. The first step of this method is to embed the experimental time series $\left\{x_{n}\right\}$ into a delay embedded state space with suitable embedding dimension $d$ and time lag $\tau$ by creating the set of delay vectors $X n=\left(x_{n}, \mathrm{x}_{n-\tau}, \ldots x_{n-(d-1) \tau}\right)$ [20]. Then the reconstructed delay vectors are transformed by using observed local linear dynamics. The transformed vectors in this embedded state space with suitable embedding parameters will be concentrated around the UPOs. Since biological systems have inherent nonstationarity associated with parameter changes that is reflected in changes in the UPO structure, the UPO structure is expected to be detectable within IEI series in a short time-window. The analysis is performed on a data window, whose width is short enough to approximate stationary system states, and long enough to accumulate good statistics at the same time. The extraction of UPOs is to look for peaks in the orbital density distribution of the transformed data. For discrete-time dynamics in a delay embedded state space, a period $p$ orbit will comprise $p$ individual points, which are related to each other through a cyclic symmetry. For example, projecting the periodic orbits in $d$-dimensional delay embedded state space into a two-dimensional space, all period- 1 orbits lie along the diagonal, all period- 2 orbits are pairs of points with reflection symmetry across the diagonal, and period-3 orbits are triplets of points with triangular symmetry. With the symmetry, the UPO hierarchy can be identified from the two-dimensional histogram of the transformed orbit density distribution. In addition, real experimental data sets are usually contaminated by mixtures of dynamical and observational noise, which make the observed sharp peaks in the distribution of transformed data blurred into broad maxima. To assess the significance of identified peaks, we used the amplitude-adjusted Fourier transform surrogates [21], which is a realization of a linear stochastic model of the data formed with the same amplitude distribution and approximately the same autocorrelation function as the original data. Therefore, we do not expect UPO structure to exist in the surrogates. By using multiple realizations of surrogate data [21] and the statistics of extremes [17], we can estimate the probability that a peak observed from our data is statistically significant. The observed UPOs were considered to be statistically significant only if the distribution densities of the transformed peaks were larger than $95 \%$ of the maximum peaks observed from 50 surrogate sets.

\section{Results}

\section{Bursting Pattern of Type A Neurons in Injured DRG}

A significantly higher presence of SMPO was detected $\left(68 / 386,18 \% ; \chi^{2}\right.$ test, $\left.p<0.01\right)$ in chronic compressed DRG neurons. The resting potential of normal DRG cells $(-9.3 \pm 6.1 \mathrm{mV}, \mathrm{n}=23)$ showed no significant difference from that of injured DRG neurons with SMPO (-56.2 \pm $1.4 \mathrm{mV}, \mathrm{n}=43)$ and that without SMPO $(-58.8 \pm$ $8.1 \mathrm{mV}, \mathrm{n}=144, \mathrm{p}>0.05)$. Of these 68 neurons, 36 neurons discharged spontaneously in bursting pattern which is characterized in most cases $(30 / 36)$ by the topological geometric features of voltage oscillations, i.e. the first spike in a burst emerged from amplitude-increasing damped SMPO and the discharge terminated by an am- 
Fig. 1. A typical example of bursting of the DRG neuron discharging spontaneously. a The raw recording of membrane potential. b The spindle-like SMPO evoking a burst sometimes or not. c A train of bursting. Occasionally a spike can be lost within a burst. All recordings are from the same neuron and the action potential in $\mathbf{b}$ and $\mathbf{c}$ are truncated.

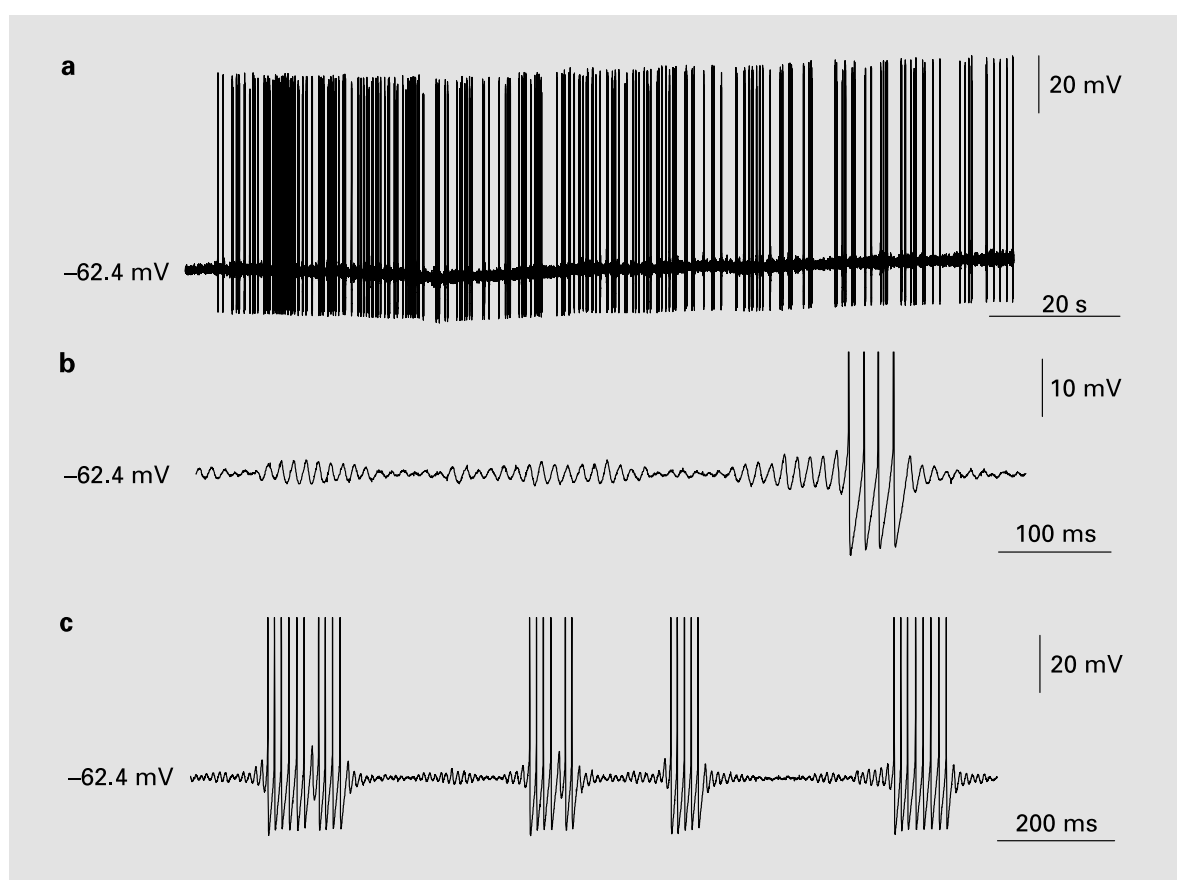

plitude-decreasing damped SMPO. The rhythm of these bursting behaviors is all irregular and on-off duty of cycle of bursts ranged from $70 \mathrm{~ms}$ to $1 \mathrm{~s}$ on and from $30 \mathrm{~ms}$ to $4 \mathrm{~s}$ off. A typical example is shown in figure 1, in which spindle-like SMPO can be found frequently. Sometimes it evokes a burst, but this is not always the case (fig. 1b). Occasionally, a spike can be lost within a burst even if the depolarization of membrane is high (fig. 1c). The intraburst frequency is relatively constant. We can find this feature in figure $2 \mathrm{a}$ and $\mathrm{b}$, in which the burst interspike intervals (BISI) are distributed around an average value of $13.3 \pm 0.9 \mathrm{~ms}$.

\section{Identification of UPOs of IEI Series}

The IEIs ranging from $63.2 \mathrm{~ms}$ to $4 \mathrm{~s}$ are extracted for UPO analyses (fig. 2a). For the above sample, we chose $40 \mathrm{~ms}$ for the discriminating threshold and the width of 128 sequential IEI data points for the data window within a relatively stationary piece of IEI series (fig. $2 \mathrm{c}$ ). It can be seen that the return map of this subset has no obvious structure. The optimal embedding parameters were chosen with embedding dimension $d=3$ and time lag $\tau=1$.

In figure $3 \mathrm{a}$, there is one strong peak along the diagonal, which indicates one significant period- 1 orbit. In figure $3 \mathrm{~b}$, four pairs of points with reflection symmetry were found, of which one is highly significant. In figure $3 \mathrm{c}$, we can enumerate three possible period-3 orbits, of which one is significant. These orbits form a complex but predictable lattice of regions in which the dynamics of the bursting occurrence is deterministic.

We identified the UPOs in 10 data sets selected respectively from the above 30 recordings in which bursting pattern similar to those of elliptic bursting type were found. All these data sets had significant period-1 orbits, $80 \%$ significant period- 2 orbits and $60 \%$ significant period-3 orbits. This suggests that UPOs exist extensively in the IEI series of bursting from the injured DRG neuron and the deterministic dynamics underlies the occurrence of bursting at least in many cases.

\section{Discussion}

Normally, the soma of an intact neuron in DRG seldom spontaneously generates discharge activity. But recently, it was found that a significantly larger proportion of neurons in injured DRG with chronic compression display spontaneous discharge in their soma. These spontaneous discharges were found to be associated with the pain pathology $[7,8,15,16]$, such as paresthesia, pain, and hyperalgesia [22]. A number of these discharges were found to be of a novel type of bursting pattern which is greatly different from that of integrate-and-fire type. Because the discharge dynamics of a neuron plays an essen- 

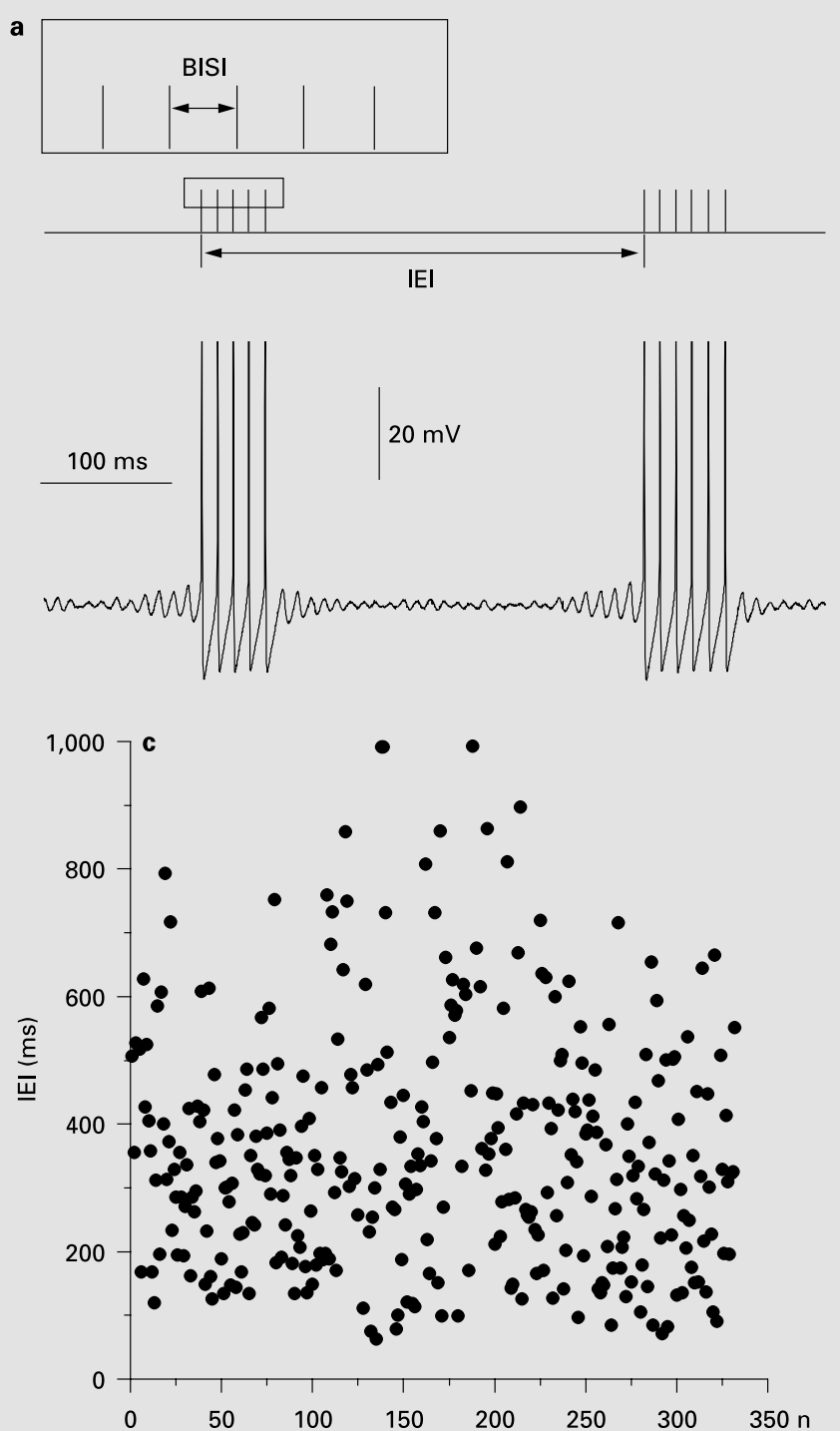
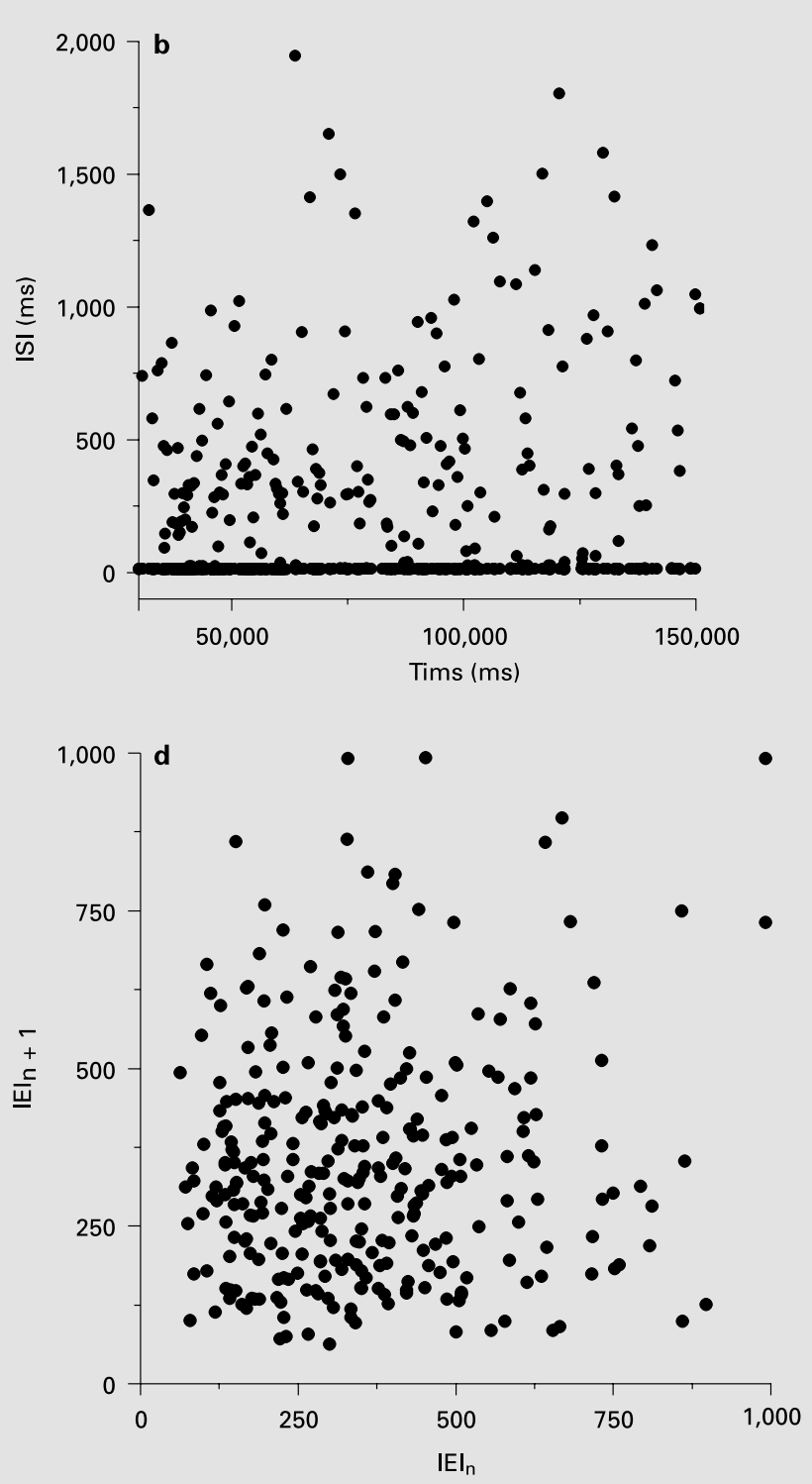

Fig. 2. Extraction of the IEIs from recording. a A piece of raw recording of membrane potential and the schematic illustration of the IEI and the BISI. b The ISI series. $\mathbf{c}$ The obtained IEI series of the windowed data subset. $\mathbf{d} \mathrm{n}$ is the index of data point. The return map between $\mathrm{IEI}_{\mathrm{n}+1}$ and $\mathrm{IEI}_{\mathrm{n}}$ of subset. The action potential in $\mathbf{a}$ is truncated.

tial role in neural coding, it is very interesting and important to elucidate the underlying dynamics of this type of spontaneous discharge. To determine the exact topological type of bursting in figure 1, a bifurcation analysis of the neuron is needed. In particular, the slow conductances need to be frozen, which is not feasible at present. We can try to determine the exact topological type of bursting using the geometry of voltage oscillations. First, the exis- tence of subthreshold membrane oscillations is a strong indicator showing the resting state is near the AndronovHopf bifurcation. The sudden jump of oscillation amplitude at the onset of bursting indicates that the bifurcation is of subcritical type, and thereby the bursting is 'subHopf $/ *$ ' type [10]. Since the intraburst frequency is relatively constant, the bifurcation from spiking state back to a resting one cannot be of saddle homoclinic type or sad- 

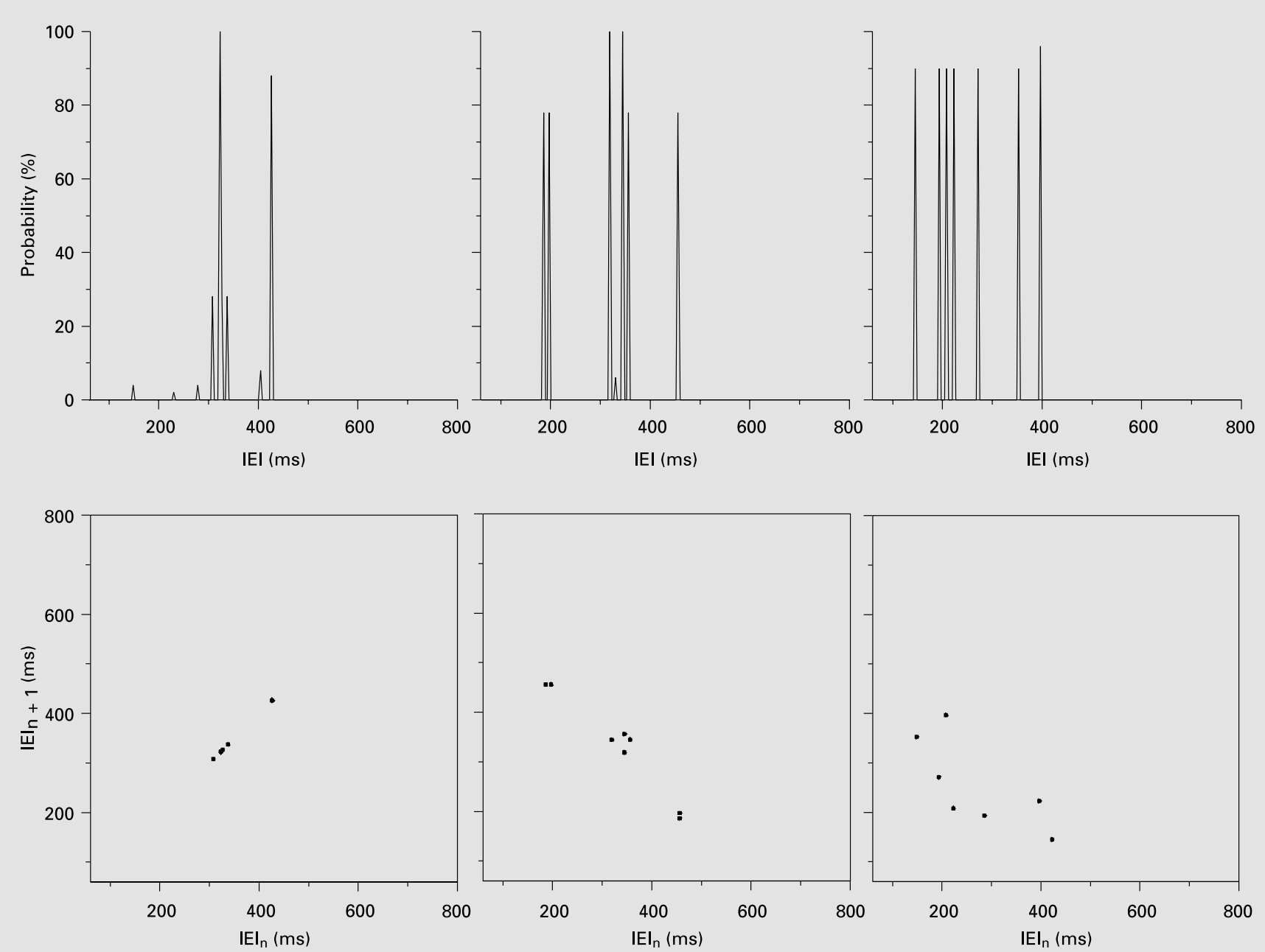

Fig. 3. Detection of UPOs in the IEI series within the window with width of 128 IEI data (top row). Probability of period-1, period-2 and period-3 transformed data density (bottom row). The density plots of period-1, period-2 and period-3 transformed data. Black points indicate that the probability of the transformed data density being outside the distribution of maximum peaks observed from 50 transformed surrogate densities is no less than $95 \%$.

dle-node on invariant circle type. Since the amplitude of spiking is also relatively constant, supercritical Andronov-Hopf bifurcation of a limit cycle is highly unlikely. Thus, the most likely bifurcation is of fold limit cycle type. Therefore, the bursting in figure 1 is most likely to be that of 'subHopf/fold cycle' type [10], also known as elliptic bursting. Since some studies have reported that the membrane potential oscillations similar to that of elliptic bursting can emerge from stochastic dynamics [11-13], the detection of their deterministic dynamics is the premise of determining the exact topological type of bursting. The statistically significant hierarchy of UPOs means that the recorded bursting comes from the deterministic dynamics with all topological geometric features of voltage oscillations of elliptic bursting.

In elliptic bursting dynamics, the Andronov-Hopf bifurcation plays an important role in the onset of bursting. It can make dynamics of the resting state of a neuron bifurcate from a focus dynamics to SMPO [23]. Compared with normal DRG, it has been noticed that a significantly larger proportion of neurons in injured DRG display a damped or sustained SMPO which has a lower rheobase [5] and can induce some rhythms [5], such as integer multiples firing [4], and bursting patterns similar 
to those in elliptic type. It has been found that some ion factors modulate the presence of SMPO [4-9, 23] and SMPO makes a neuron sensitive to the precise timing of input pulses. This is essential for selective communication with other neurons via resonant bursting [24]. Based on this mechanism of selective communication, in injured DRG cases, a neuron exhibiting damped or sustained SMPO also has perhaps selective response to the temporal structure of afferent spike trains. In addition, although it has been reported that the resting potential level has no significant difference between neurons in normal DRG and those in injured DRG [5], we believe that responsiveness of the silent neurons in injured DRG has yet to be investigated. The resting state of some of them is also likely to possess focus dynamics which can make a neuron sensitive to the fine temporal structure of afferent spike trains [2].

In some other studies, the bursting patterns similar to those in the elliptic type were found [25-27]. After the bursting initiates from amplitude-growing SMPO, it has full-amplitude spikes, and ISIs show no observable increase at the bursting termination followed by an amplitude-decreasing SMPO. This feature differs largely from that of integrate-and-fire type. Since many conductancebased simulation models, such as those of Morris and Lecar [27], can display bursting of integrate-and-fire type or elliptic type (whether it is integrate-and-fire type or elliptic type depends on the value of parameters), we can deduce that some factors in a neuron modulate the topological type of bursting dynamics of the neuron. But which factors in an injured neuron that can switch bursting of integrate-and-fire type to elliptic bursting has yet to be further probed. Based on the marriage of theoretical analysis and experimental investigation, the identification of the bursting mechanism in experimental conditions is the first step to the discovery of what factors switch bursting of integrate-and-fire type to elliptic bursting.

\section{Conclusion}

By using a UPOs extraction method, statistically significant unstable period-1, period-2 and period-3 orbits were extracted from the IEI series of bursting recorded from the injured DRG neuron. The results indicate that deterministic dynamic behaviors significantly underlie the occurrence of bursting from the type A neurons in the injured DRG. Based on this result and the exact topological type of deterministic dynamics of bursting determined by using the geometry of voltage oscillations, the elliptic bursting of neuron in injured DRG with chronic compression is identified.

\section{Acknowledgements}

This study was supported by China National Foundation of Natural Sciences grant (30030040) and the National Basic Research Program (G1999054000) of China.

\section{References}

1 Izhikevich EM: Subcritical elliptic bursting of Bautin type. SIAM J Appl Math 2000;60:503535.

2 Izhikevich EM: Resonate-and-fire neurons. Neural Netw 2001;14:883-894.

3 Christopher A, Negro D, Hsiao CF, Chandler $\mathrm{SH}$, Garfinkel A: Evidence for a novel bursting mechanism in rodent trigeminal neurons. Biophys J 1998;75:174-182.

4 Xing JL, Hu SJ, Xu H, Han S, Wan YH: Subthreshold membrane oscillations underlying integer multiples firing from injured sensory neurons. Neuroreport 2001;12:1311-1313.

5 Xing JL, Hu SJ, Long KP: Subthreshold membrane potential oscillations of type A neurons in injured DRG. Brain Res 2001;901:128136.
6 Amir R, Liu CN, Kocsis JD, Devor M: Oscillatory mechanism in primary sensory neurons. Brain 2002;125:421-435.

7 Liu CN, Dever M, Waxman SG, Kocsis JD: Subthreshold oscillations induced by spinal nerve injury in dissociated muscle and cutaneous afferents of mouse DRG. J Neurophysiol 2002;87:2009-2017.

8 Liu CN, Michaelis M, Amir R, Dever M: Spinal nerve injury enhances subthreshold membrane potential oscillations in DRG neurons: Relation to neuropathic pain. J Neurophysiol 2000;84:205-215.

9 Amir R, Michaelis M, Devor M: Burst discharge in primary sensory neurons: Triggered by subthreshold oscillation, maintained by depolarizing afterpotentials. J Neurosci 2002;22: 1187-1198.

10 Izhikevich EM: Neural excitability, spiking and bursting. Int $\mathbf{J}$ Bifurcat Chaos 2000;10: 1171-1266.
$11 \mathrm{Gu}$ HG, Yang MG, Li L, Liu ZQ, Ren W: Experimental observation of the stochastic bursting caused by coherence resonance in a neural pacemaker. Neuroreport 2002;13:16571660 .

12 Makarov VA, Nekorkin VI, Velarde MG: Spiking behavior in a noise-driven system combining oscillatory and excitatory properties. Phys Rev Lett 2001;86:3431-3434.

13 Yang ZQ, Lu QS, Gu HG, Ren W: Integer multiple spiking in the stochastic Chay model and its dynamical generation mechanism. Phys Lett A 2002;299:499-506.

$14 \mathrm{Hu}$ SJ, Xing JL: An experimental model for chronic compression of dorsal root ganglion produced by intervertebral foramen stenosis in the rat. Pain 1998;77:15-23. 
15 Song XJ, Hu SJ, Greenquist KW, Zhang JM, LaMotte RH: Mechanical and thermal hyperalgesia and ectopic neuronal discharge after chronic compression of dorsal root ganglia. $\mathrm{J}$ Neurophysiol 1999;82:3347-3358.

16 Bair W, Koch C, Newsome W, Britten K: Power spectrum analysis of bursting cells in area MT in the behaving monkey. J Neurosci 1994; 14:2870-2892.

17 So P, Francis JT, Netoff TI, Gluckman BJ, Schiff SJ: Periodic orbits: A new language for neuronal dynamics. Biophys J 1998;74:27762785 .

18 So P, Ott E, Schiff SJ, Kaplan DT, Sauer T, Grebogi C: Detecting unstable periodic orbits in chaotic experimental data. Phys Rev Lett 1996;76:4705-4708.
19 So P, Ott E, Sauer T, Gluckman BJ, Grebogi C, Schiff SJ: Extracting unstable periodic orbits from chaotic time series data. Phys Rev E 1997;55:5398-5417.

20 Sauer T: Reconstruction of dynamical systems from interspike intervals. Phys Rev Lett 1994; 72:3811-3814.

21 Theiler J: Two tools to test time series data for evidence of chaos and/or nonlinearity. Integr Physiol Behav Sci 1994;29:211-216.

22 Devor M, Seltzer Z: Pathophysiology of damaged nerves in relation to chronic pain; in Wall PD, Melzack R (eds): Textbook of Pain, ed 4. London, Churchill Livingstone, 1999, pp 129164.

23 White JA, Budde T, Kay AR: A bifurcation analysis of neuronal subthreshold oscillations Biophys J 1995;69:1203-1217.
24 Izhikevich EM, Desai NS, Walcott EC, Hoppensteadt FC: Bursts as a unit of neural information: Selective communication via resonance. Trends Neurosci 2003;26:161-167.

25 Pedroarena CM, Pose IE, Yamuy J, Michael HC, Morales FR: Oscillatory membrane potential activity in the soma of a primary afferent neuron. J Neurophysiol 1999;82:1465-1476.

$26 \mathrm{Wu} \mathrm{NP}$, Hsiao CF, Chandler SH: Membrane resonance and subthreshold membrane oscillations in mesencephalic V neurons: Participants in burst generation. J Neurosci 2001;21:37293739 .

27 Morris C, Lecar H: Voltage oscillations in the barnacle giant muscle fiber. Biophys J 1981;35: 193-213. 